Research Article

\title{
Computational Modeling of the Interaction of Silver Nanoparticles with the Lipid Layer of the Skin
}

\author{
Andrea Fabara, ${ }^{1}$ Sebastián Cuesta $\mathbb{D}^{2},{ }^{2}$ Fernanda Pilaquinga, ${ }^{3,4}$ and Lorena Meneses $\mathbb{D}^{2}$ \\ ${ }^{1}$ Faculty of Chemistry, University of Strasbourg, Strasbourg, France \\ ${ }^{2}$ Laboratory of Computational Chemistry, Pontificia Universidad Católica del Ecuador, Quito, Ecuador \\ ${ }^{3}$ Laboratory of Nanotechnology, Pontificia Universidad Católica del Ecuador, Quito, Ecuador \\ ${ }^{4}$ Department of Chemistry, University of the Balearic Islands, Palma de Mallorca, Balearic Islands, Spain \\ Correspondence should be addressed to Lorena Meneses; lmmeneses@puce.edu.ec
}

Received 6 February 2018; Revised 16 April 2018; Accepted 23 April 2018; Published 4 June 2018

Academic Editor: Rachid Seqqat

Copyright $\odot 2018$ Andrea Fabara et al. This is an open access article distributed under the Creative Commons Attribution License, which permits unrestricted use, distribution, and reproduction in any medium, provided the original work is properly cited.

\begin{abstract}
Silver nanoparticles are recognized for numerous physical, biological, and pharmaceutical applications. Their main uses in the medical field comprise diagnostic and therapeutic applications. In this project, the interaction between silver nanoparticles and the lipid layer of the skin was studied in order to know how nanoparticles behave when they are in contact with the skin. Energies of the silver nanoparticles were calculated through the optimization of silver clusters using density functional theory implemented in the Gaussian program 09W. Biological molecules such as glucose, stearic acid, palmitic acid, and quercetin present in coated nanoparticles and in the skin were also optimized. The silver clusters containing 6 atoms were proven to be the most stable complexes. Moreover, a study of molecular orbital describing HOMO interactions of the clusters was performed showing that the electronic density was around the silver cluster. Molecular dynamics simulation was performed using Abalone program. Silver nanoparticles seemed to have very good clearance properties in our molecular dynamics simulation because over a certain period of time, the silver cluster got far away from the biological molecules.
\end{abstract}

\section{Introduction}

Silver nanoparticles have unique optical, electrical, and thermal properties. Present applications range from photovoltaics cells to biological and chemical sensors. Silver nanoparticles also present biological applications such as molecular diagnostics, photonic devices, nanomedicine drug delivery, imaging diagnostics, and biosensing $[1,2]$. However, it is important to be sure about the possible consequences of their use.

Nanoparticles are being daily used, yet a concern rises due to the fact that their toxicity could put human health at risk [3]. Thus, to develop new nanoparticles and continue their use and development, more studies and evaluation have to be done. One of the possibilities in doing so is by investigating silver nanoparticles through quantum mechanics calculations.

Computational modeling is a powerful tool compared to experimental limitations. In a computational work, it is possible to manage each parameter separately and identify the mechanism responsible for the experimental result. With a computational study, it is feasible to simulate interactions under different conditions that are not always possible to be studied in the lab [4]. All nanomaterials are size dependent because their physical and chemical properties will depend on the size of the nanoparticle. The composition, size, shape, and environment of nanoparticles can strongly influence their eventual application as they have excellent properties for antimicrobial coatings, textiles, keyboards, wound dressings, and biomedical devices $[5,6]$.

Theoretical studies on the affinity of silver ions with DNA at a molecular level were performed to determine the interaction of silver ions with a cytosine base and an adenosine base, using ab initio calculations and density functional theory (DFT) [7]. Also, a chemical reactivity analysis of biological molecules in the presence of silver ions in the DFT framework has been performed in order to find the molecular structure with the lowest energy, molecular orbitals, and chemical reactivity parameters $[8]$. 
Lipids are fundamental structural components of cell membranes. They are little oxidizable molecules serving as an energy reservoir for the cell. Lipids are key components of the skin cell membrane that need to be analyzed because when there is an attack from foreign agents, they become injured [9]. Lipids analyzed in this research were two saturated lipids, palmitic and stearic acids, present in the lipid layer of the skin . Stearic acid was chosen for the study because it was the second most common lipid after palmitic acid contributing to prove the stability of the silver complexes.

In this study, the interaction between silver nanoparticles and the lipid layer was investigated. Since an organic crystal structure is not available, a computational study was performed to evaluate the compounds and to determine structural information. This research aims to establish a quantitative structure-activity between the interaction of silver nanoparticles and the lipid layer of the skin (palmitic and stearic acids).

In previous works, small silver clusters were studied using DFT methods. Poteau and his team had studied the ground-state geometries and the binding energies per atom of small silver clusters, from 4 to 8 silver atoms. With their results, they managed to parameterize distance-dependent tight-binding Hamiltonian and accomplished good correlation between their model and ab initio calculations [10]. Another DFT study was done on small anionic, neutral, and cationic silver cluster hydrides using the PW91PW91 method. Here, binding energies showed different behaviors depending on the number of silver atoms; cationic clusters are the ones with lowest binding energies in clusters with an odd number of silver atoms while neutral clusters are the ones with an even number of silver atoms [11].

In other studies, silver nanoparticles coated with glucose and quercetin (a flavonoid) have been reported as green synthesis methodologies. Coated silver nanoparticles with Piper aduncum presented extraordinary healing properties $[12,13]$. Quercetin, being the principal component of Piper aduncum, was chosen to interact with the silver clusters. Silver nanoparticles covered with quercetin proved to have therapeutic properties in addition to the antibacterial properties. However, silver nanoparticles covered with glucose did not show any healing characteristics except for antibacterial properties of the silver nanoparticles $[12,14,15]$. Thanks to their small size, large surface area, and versatile physicochemical properties, these coated silver nanoparticles could enter cells and organelles leading to the possibility of nanomedicine [15]. Therefore, we also proposed to determine the stability of these complexes, since the silver nanoparticles can be released once they have completed their therapeutic action.

\section{Materials and Methods}

The geometry optimization of silver clusters was carried out at the B3LYP/LANL2DZ level of theory, while palmitic acid, stearic acid, glucose, and quercetin geometries were carried out at the B3LYP/6-311G level of theory, implemented in the Gaussian 09W package of programs [16]. A computational modeling of silver clusters with the biological

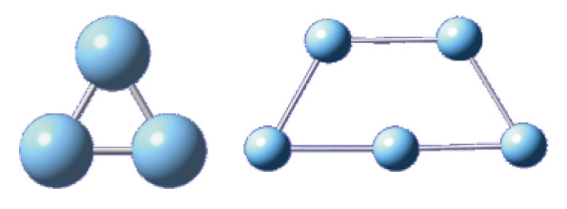

(a)

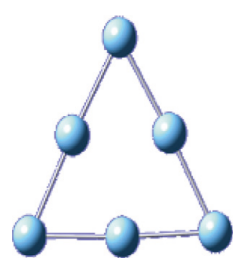

(c)
FIGURE 1: Silver cluster optimized and conserved: (a) cluster of 3 silver atoms, (b) cluster of 5 silver atoms, and (c) cluster of 6 silver atoms.

molecules was analyzed, and lower energy conformations were identified. The energy of the cluster interaction was calculated using the following equation:

$$
\Delta I=E_{(\mathrm{Agc}-\mathrm{BM})}-E_{\mathrm{Agc}}-E_{\mathrm{BM}},
$$

where $\Delta I=$ interaction energy, $E_{(\mathrm{Agc}-\mathrm{BM})}=$ energy of the cluster-biomolecule complex, $E_{\mathrm{Agc}}=$ energy of the silver cluster, and $E_{\mathrm{BM}}=$ energy of the biomolecule.

Also, a study of the MO theory describing HOMO interactions of clusters was carried out.

Molecular dynamics using the Abalone program (a molecular simulation program with AMBER-ii force field) was performed to see how molecules interact for a given period of time (from 5 to $20 \mathrm{ps)} \mathrm{[17].} \mathrm{To} \mathrm{carry} \mathrm{out} \mathrm{the}$ dynamics, a .pdb file with the optimized geometry of the cluster-biomolecule complex obtained from the DFT study was opened in the Abalone program. The option of molecular dynamics was selected; working conditions were set at $1 \mathrm{~atm}$ of pressure, $298.15 \mathrm{~K}$ of temperature, $2.0 \mathrm{fs}$ time per step, and 5 or 20 ps of duration.

\section{Results and Discussion}

The methods within the framework of the density functional theory (DFT) were used to optimize the structure of the silver nanoparticles and also the stearic acid, palmitic acid, quercetin, and glucose. Vibrational frequencies at the ground state were calculated in order to ensure that they were at a minimum level.

3.1. Energetic Study. Different geometries and number of atoms were tested to form a silver nanoparticle. It took many tries using different numbers of silver atoms $(3,13,30,50$, $64,85,100,120$, etc.) to form the silver nanoparticle with different geometries and shapes. However, the silver clusters were not correctly optimized because of the loss of geometry.

From all the geometries, the silver clusters with 3, 5, and 6 silver atoms were chosen to continue the study. Figure 1 shows the clusters of silver nanoparticles that were optimized and where sizes and geometries were conserved.

Once the silver clusters were optimized, the biological molecules were optimized as well. Figure 2 illustrates biological molecules that were analyzed: lipids such as palmitic acid and stearic acid, quercetin which is a flavonoid, and glucose a sugar.

Interaction between biological molecules (quercetin, glucose, palmitic acid, and stearic acid) and the three silver 


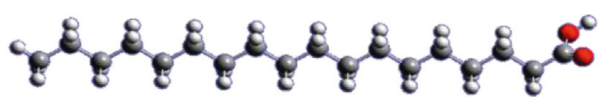

(a)

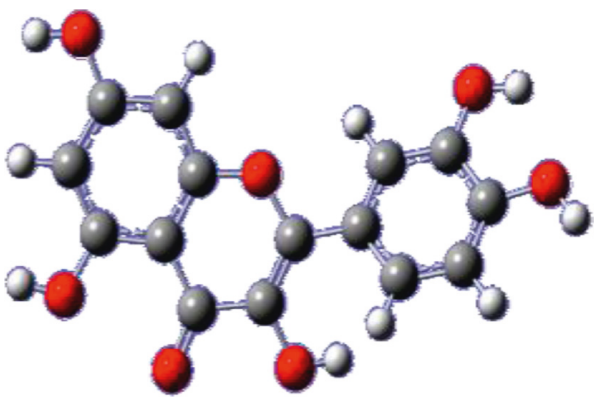

(c)

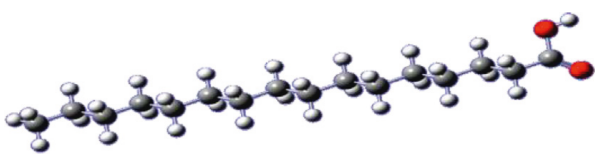

(b)

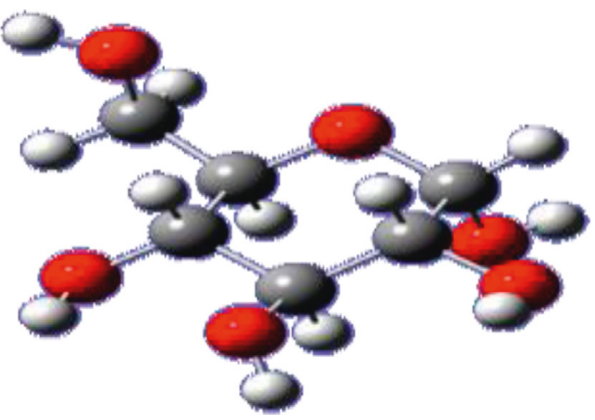

(d)

FIgURE 2: Biological molecules used in this study: (a) stearic acid, (b) palmitic acid, (c) quercetin, and (d) glucose.

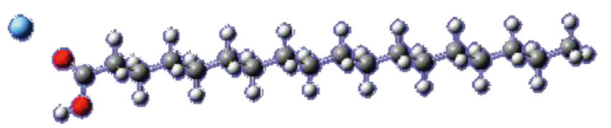

(a)

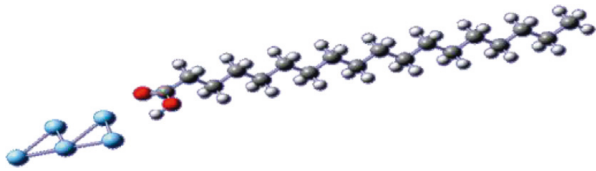

(c)

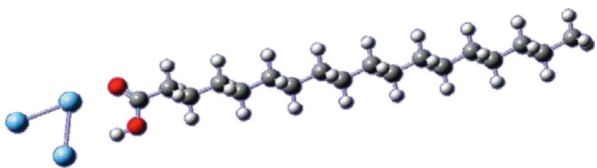

(b)

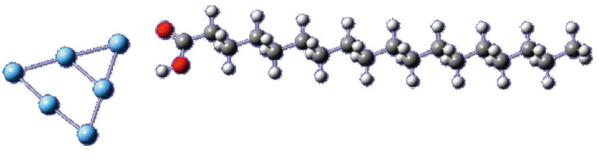

(d)

Figure 3: Optimized geometries of stearic acid with (a) a single silver atom, (b) cluster of 3 silver atoms, (c) cluster of 5 silver atoms, and (d) cluster of 6 silver atoms.

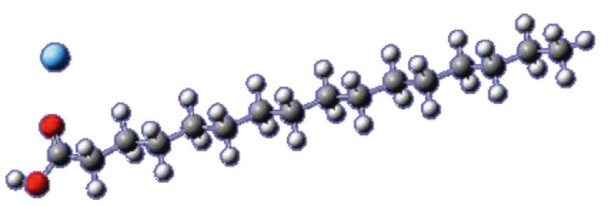

(a)

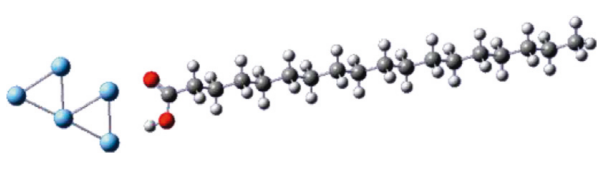

(c)

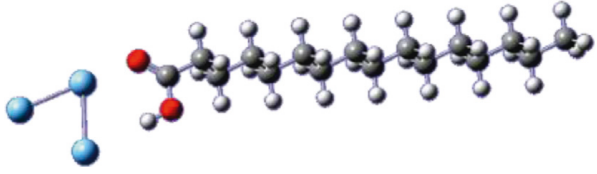

(b)

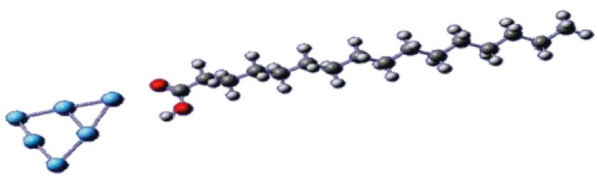

(d)

Figure 4: Optimized geometries of palmitic acid with (a) a single silver atom, (b) cluster of 3 silver atoms, (c) cluster of 5 silver atoms, and (d) cluster of 6 silver atoms.

clusters previously optimized was simulated. The optimized geometries of the interaction between the biomolecules with a single silver atom are also shown so as to find the differences between these and the cluster interactions. All of them were optimized in order to know if they could form a complex and to measure the distance of the cluster and the biomolecule. Based on the information provided by $\mathrm{He}$ and Zeng [18], the silver atom and the clusters were introduced in a position in which they were close to the carboxyl group which is the hydrophilic part of the fatty acids, as shown in Figures 3 and 4 . In this conformation, the complexes presented the lowest energy.

In Figures 5 and 6, the most stable complex between the silver atom and clusters with glucose and quercetin is shown. In this case, it can be seen that the clusters are close to the oxygen atoms of the molecules, also being the hydrophilic 


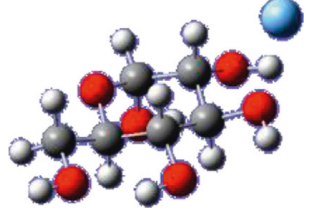

(a)

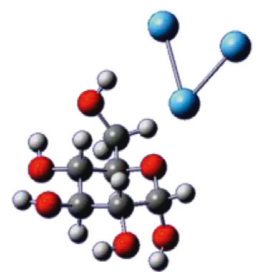

(b)
FIGURE 5: Optimized geometry of glucose with (a) a single silver atom and (b) the cluster of 3 silver atoms.
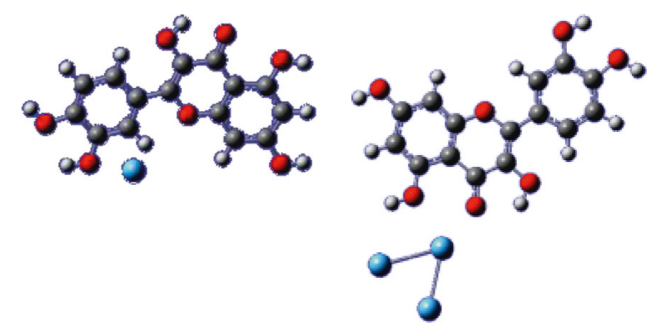

(a)

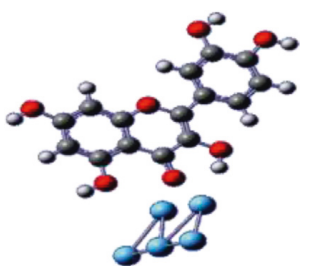

(c)

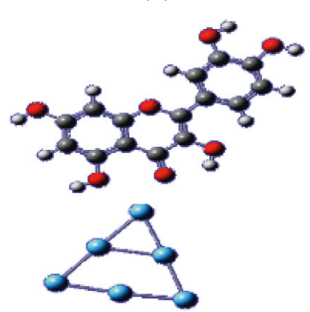

(d)

FIGURE 6: Optimized geometries of quercetin with (a) a single silver atom, (b) cluster of 3 silver atoms, (c) cluster of 5 silver atoms, and (d) cluster of 6 silver atoms.

parts. With glucose, it was not possible to obtain optimized geometries with 5 and 6 silver atom clusters.

In previous studies of reactivity analysis, it was found that the main interaction between palmitic acid and the silver ion was through the carboxylic acid of the lipid [8]. Besides, the interaction between biological molecules and silver clusters was through the hydroxyl groups as compared with the study between PVA and Ag55 nanocrystals [19]. Silver nanoparticles go through shape-dependent interaction with the lipid layer. While these interactions are being studied, these nanoparticles are being used more and more for their antibacterial and antimicrobial activities [20].

The interaction energies of the systems were calculated using (1) and are presented in Table 1.

The stabler structures are the ones with smaller energies; therefore, clusters of 6 silver atoms are considered as the most stable ones. On the contrary, when comparing these results with the ones obtained with a single silver atom, it could be seen that the systems with a single silver atom were the ones with the highest interaction energies. The different results obtained from the different clusters studied give a clear view of interaction energies, and therefore, the properties of the silver nanoparticles are size dependent.

TABLE 1: Interaction energies between the clusters of $1,3,5$, and 6 atoms of silver, stearic acid, palmitic acid, and quercetin.

\begin{tabular}{lc}
\hline System & $\Delta I(\mathrm{kcal} / \mathrm{mol})$ \\
\hline Stearic acid $1 \mathrm{Ag}$ & -2.79 \\
Stearic acid cluster $3 \mathrm{Ag}$ & -18.58 \\
Stearic acid cluster $5 \mathrm{Ag}$ & -8.51 \\
Stearic acid cluster 6 Ag & -75.47 \\
Palmitic acid 1 Ag & -3.41 \\
Palmitic acid cluster 3 Ag & -18.65 \\
Palmitic acid cluster 5 Ag & -8.53 \\
Palmitic acid cluster 6 Ag & -75.43 \\
Quercetin 1 Ag & -0.81 \\
Quercetin cluster 3 Ag & -24.44 \\
Quercetin cluster 5 Ag & -12.53 \\
Quercetin cluster 6 Ag & -80.16 \\
Glucose 1 Ag & -26.50 \\
\hline
\end{tabular}

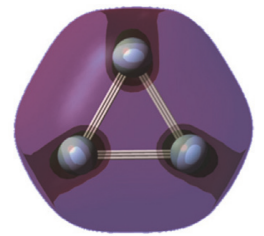

(a)

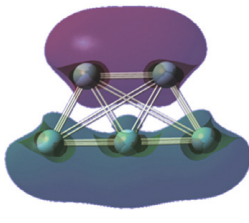

(b)

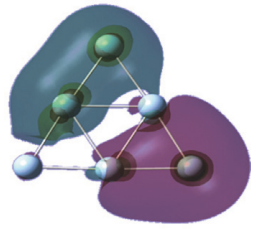

(c)
Figure 7: HOMO surfaces of clusters of (a) 3 silver atoms, (b) 5 silver atoms, and (c) 6 silver atoms.

According to our study, a silver nanoparticle with the size of our cluster of 6 silver atoms will have better properties due to its increased stability compared to small nanoparticles. The interaction energies between the metal clusters and the biological molecules suggest that nanoparticles will favorably bind to proteins and can be adsorbed with biomolecules as melamine $[21,22]$. The energies also confirm that noncoated silver nanoparticles are less stable, thus presenting bigger energies. The silver cluster covered with the biological molecule was more stable than the silver nanoparticle alone. This was favorable because it improved bioavailability, as previously demonstrated by Kumar and Randhawa [23].

3.2. Molecular Orbital Study. Molecular orbitals are regions of space that contain the electron density defined by mathematical functions that describe the wave behavior that electrons can have on molecules. These functions can be used to calculate chemical and physical properties such as the probability of finding an electron in a region of space. The border molecular orbitals HOMO and LUMO (highest occupied molecular orbital and lowest unoccupied molecular orbital, resp.) allow for knowing the regions of space where the valence electrons are, that is, those that participate in bond formations. In this way, a study of border molecular orbitals allows us to see if there is a bond and where it is located.

In this investigation, a study of molecular orbitals (MO) describing HOMO interaction of the clusters and the biomolecules was carried out in order to know if there is a bond between the biomolecules and the clusters. In Figures 7-10, 


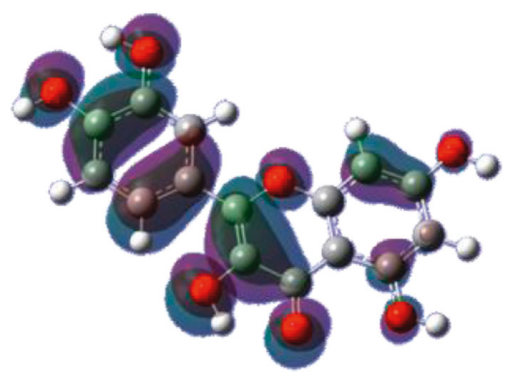

(a)

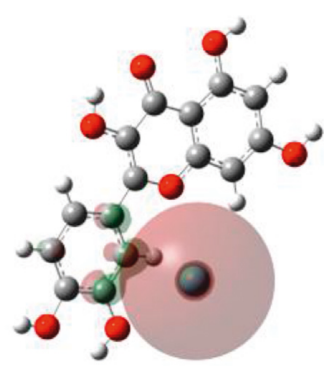

(b)

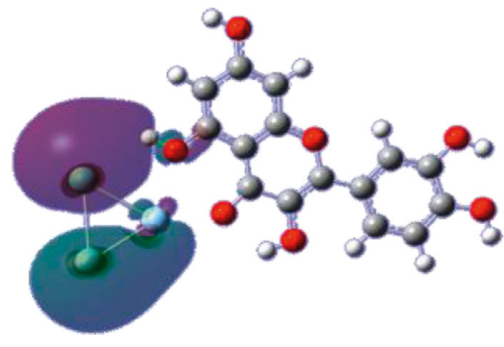

(c)

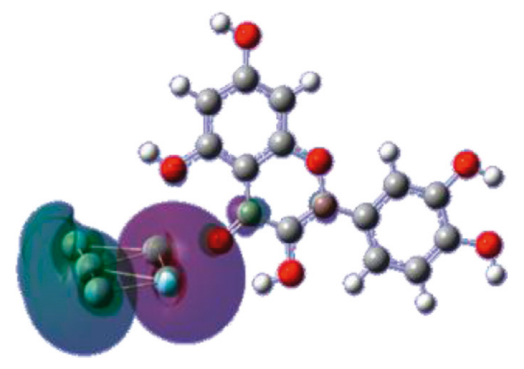

(d)

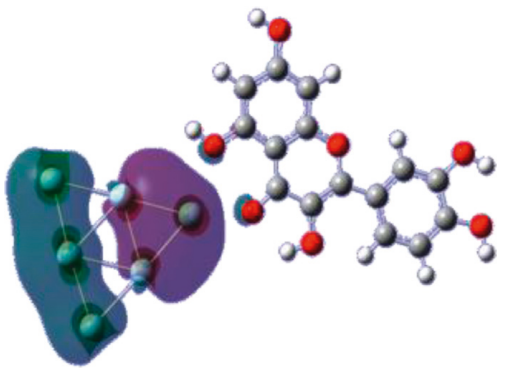

(e)

Figure 8: HOMO surfaces of (a) quercetin and the complex between quercetin with (b) a single silver atom, (c) cluster of 3 silver atoms, (d) cluster of 5 silver atoms, and (e) cluster of 6 silver atoms.

the red shapes correspond to bonding molecular orbitals, while the green shapes correspond to antibonding ones. Figure 7 illustrates the spatial and energetic properties of electrons that form orbitals in the silver clusters.

The shapes of the molecular orbitals from Figures 8-10 show that, in the stearic acid, palmitic acid, and quercetin, the energies surrounded the entire molecule. However, once the silver cluster was added, the molecular orbital energies changed and they were then located mainly around the cluster of $1,3,5$, or 6 silver atoms.

In Figure 8(a), it can be seen that the energy of the HOMO is located completely around the quercetin molecule. However, when the single silver atom and the clusters of 3,5, and 6 silver atoms are found near the quercetin molecule (Figures $8(\mathrm{~b})-8(\mathrm{e})$ ), the energy moves almost completely to the silver atoms, leaving small orbital regions around the oxygen atoms near the cluster. This indicates that there is a weak interaction between the cluster and the biomolecule. Given the simplicity of the study model, this can be considered as a bonding interaction.

In Figure 9(a), the energy of the HOMO is located completely around the stearic acid. When a single silver atom and the clusters of 3,5, and 6 silver atoms are near the biomolecule (Figures 9(b)-9(e)), the energy again moves to the silver atoms, leaving small orbital regions around the oxygen atoms of the carboxylic acid.

This indicates that there is a weak interaction between the cluster and the biomolecule. With palmitic acid, the behavior is similar, as can be seen in Figure 10.

3.3. Molecular Dynamics Study. Molecular dynamics is a powerful tool in molecular modeling because it gives the opportunity to follow and understand the structure and

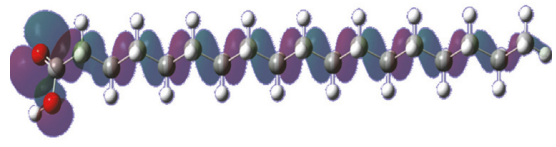

(a)

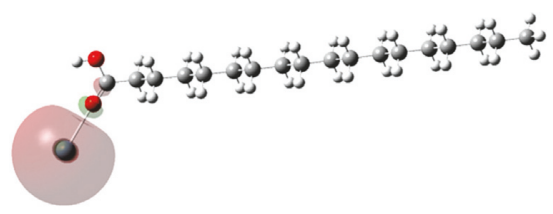

(b)

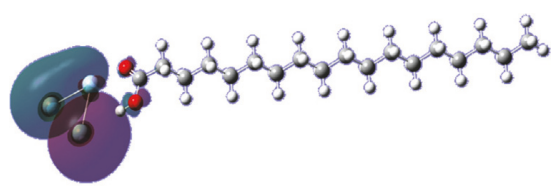

(c)

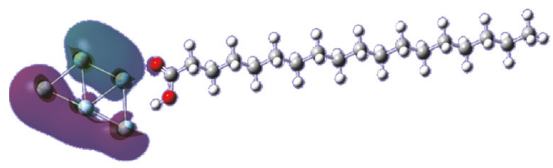

(d)

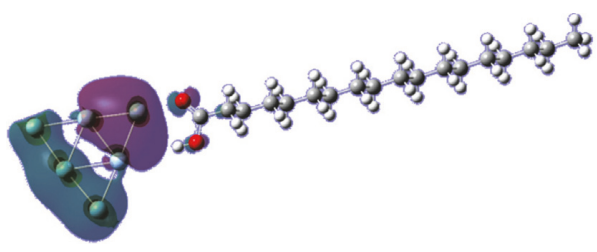

(e)

FIgURE 9: HOMO surfaces of (a) stearic acid and the complex between stearic acid with (b) a single silver atom, (c) cluster of 3 silver atoms, (d) cluster of 5 silver atoms, and (e) cluster of 6 silver atoms. 


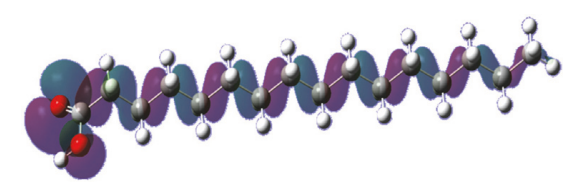

(a)

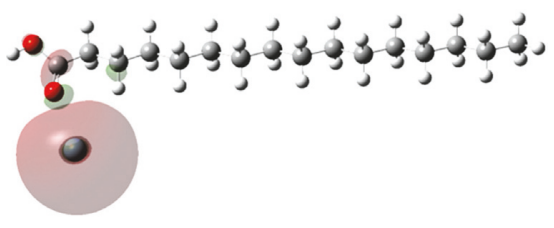

(b)

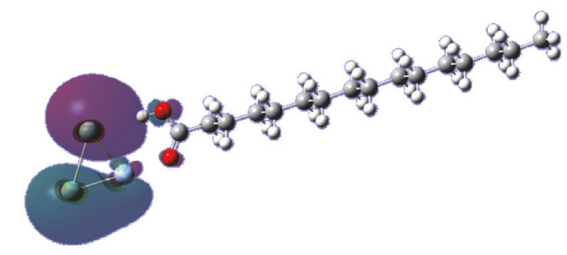

(c)

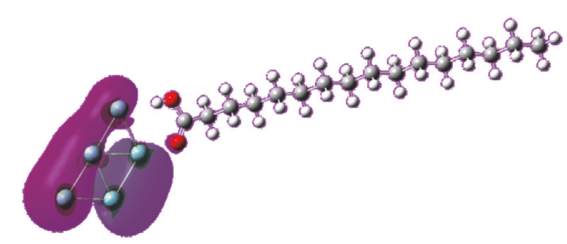

(d)

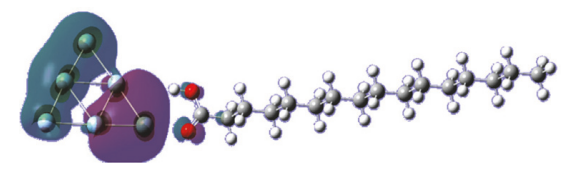

(e)

FIgURE 10: HOMO surfaces of (a) palmitic acid and the complex between palmitic acid with (b) a single silver atom, (c) cluster of 3 silver atoms, (d) cluster of 5 silver atoms, and (e) cluster of 6 silver atoms.

dynamics of molecules [24]. The Abalone program is used to simulate the natural motion of biological macromolecules. Silver nanoparticles have many interesting properties as previously mentioned. Nevertheless, silver nanoparticles reveal an extended retention in an organism which is cause for concern due to toxicity. For that reason, coated (Piper aduncum) silver nanoparticles with optimal clearance characteristics are very important because they minimize toxicity [12].

Furthermore, the Abalone program contributed by conducting the simulations of molecular dynamics in order to determine the interactions of biological molecules and the silver clusters for a fixed period of time, 5 and 20 ps. The molecules were allowed to move giving a view of the dynamical evolution of the system. At different times, distances increased between the cluster and the biological molecule as indicated in Figures 11-13.

Distances from the oxygen of the carboxylic acid to the first atom of the silver cluster were measured. The distance

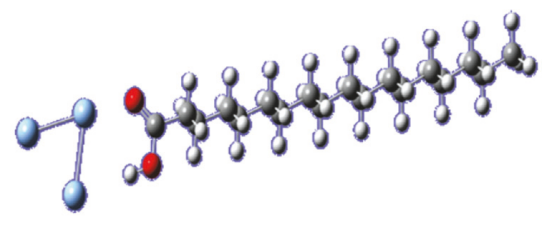

(a)
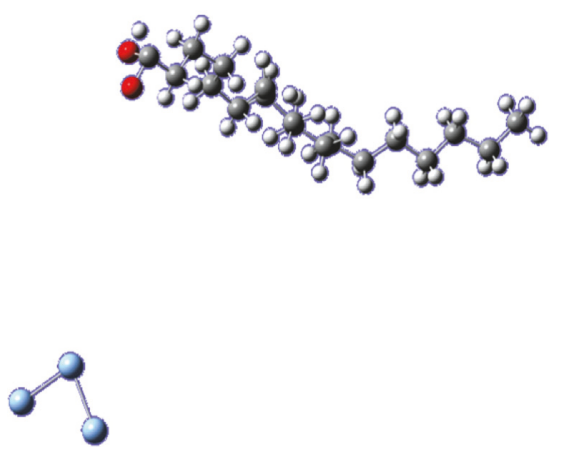

(b)
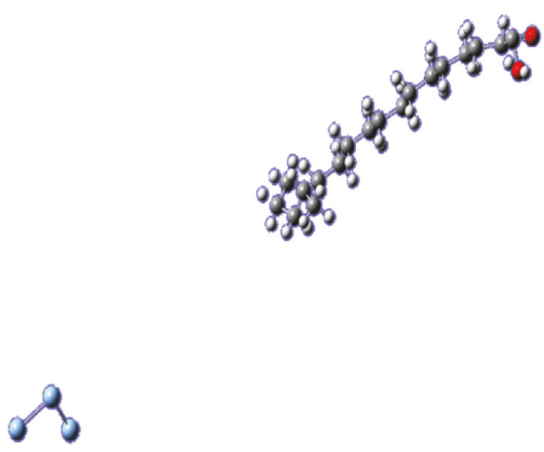

(c)

FIgURE 11: Molecular dynamics performed at 298.15 K. Stearic acid with 3 silver atoms cluster at (a) 0 ps, (b) 5 ps, and (c) 20 ps.

between stearic acid and the cluster of 3 silver atoms at the beginning of the dynamic was $2.33 \AA$. After 5 ps, the distance increased to $10.09 \AA$, and after 20 ps, it changed to $32.67 \AA$. Palmitic acid and the different clusters of 3,5, and 6 silver atoms followed the same path as stearic acid. This meant that the cluster was moving away, while the distance kept increasing. These results indicate that the silver nanoparticles can go through the lipid layer of the skin, but they can also be easily separated reducing their toxicity.

In the case of the glucose, measured distances were calculated from the oxygen of the cycle to the first atom of the silver cluster. In Figure 12, the distances changed from $3.35 \AA$ at the beginning of the dynamic to $61.53 \AA$ after 20 ps. This shows that glucose does not have a strong interaction with the 3-atom silver cluster after a short period of time, and conversely, the silver cluster rapidly goes far away from the biomolecule, showing good clearance properties.

In Figure 13, distances were measured from the carbonyl oxygen of the quercetin to the first atom of the silver cluster. In the 3-atom silver cluster, the distances changed from 2.34 to $7.87 \AA$ at the end of 20 ps. With the 5 -atom silver cluster, the distances were higher, changing from 2.37 to $34.08 \AA$ 


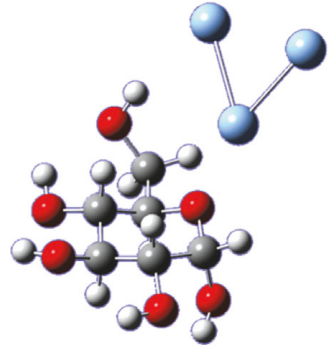

(a)

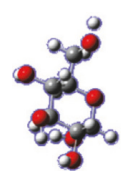

(b)

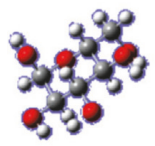

(c)

Figure 12: Molecular dynamics performed at $298.15 \mathrm{~K}$. Glucose with 3-atom silver cluster at (a) 0 ps, (b) 5 ps, and (c) 20 ps.

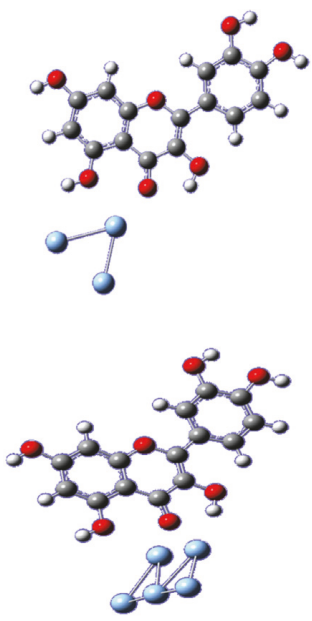

(a)
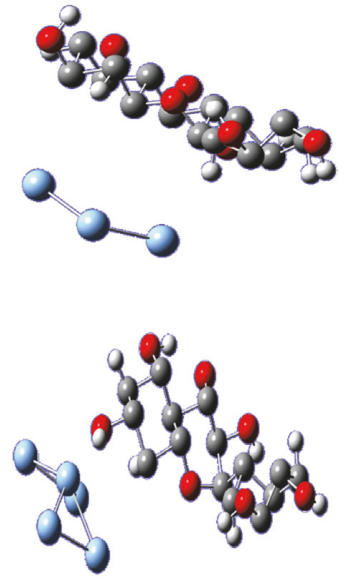

(b)
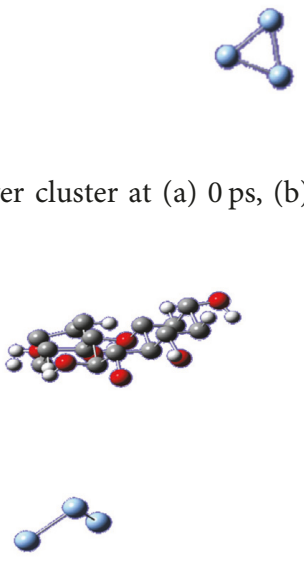

FIGURE 13: Molecular dynamics performed at 298.15 K. Quercetin with 3-and 5-atom silver clusters at (a) 0 ps, (b) 5 ps, and (c) 20 ps.

after the same period of time. This means that with small nanoparticles, there are major interactions between the nanoparticle and the biomolecule, and there is not a good clearance after short periods of time. On the contrary, with bigger nanoparticles, there is really great separation with the quercetin, showing excellent clearance properties. With the 6 -atom silver cluster, the trend was the same as the 5-atom silver cluster [13].

To probe that the complex silver cluster-stearic acid has a bonding interaction, which was broken after $5 \mathrm{ps}$, a $\mathrm{MO}$ study on the molecular dynamics structures was done. In Figure 14, it can be seen that at $0 \mathrm{ps}$, the HOMO was located around the silver cluster, but a small shape is around the oxygen of the carboxilic part of the fatty acid. When the molecular dynamic moves to $5 \mathrm{ps}$, the HOMO was located completely around the silver cluster, and at $20 \mathrm{ps}$, this was maintained. This shows that there was an orbital interaction between the stearic acid and the silver cluster when they were $2.33 \AA$ away from each other. The complex was broken when the distance increased to $10.09 \AA$ and further.

In the case of the quercetin with 3-atom silver clusters (Figure 15), it can be seen that at $0 \mathrm{ps}$, the HOMO was located around the silver cluster and a small part above the oxygen of the hydroxyl group. When the molecular dynamics moves to $5 \mathrm{ps}$, the HOMO was located completely around the silver cluster, and at $20 \mathrm{ps,} \mathrm{some} \mathrm{of} \mathrm{the} \mathrm{orbital}$ energy is again located at the biomolecule. This shows that there was an orbital interaction between the quercetin and the silver cluster when they have around $2.34 \AA$ of separation distance. The complex was broken when the distance increased. Finally, at $7.87 \AA$, the interaction returned although the molecules were away from each other. This confirms that with small nanoparticles, there are major 


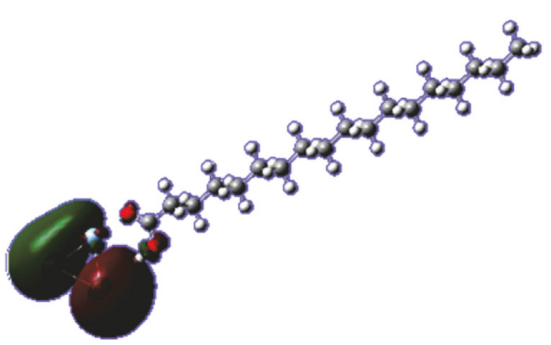

(a)
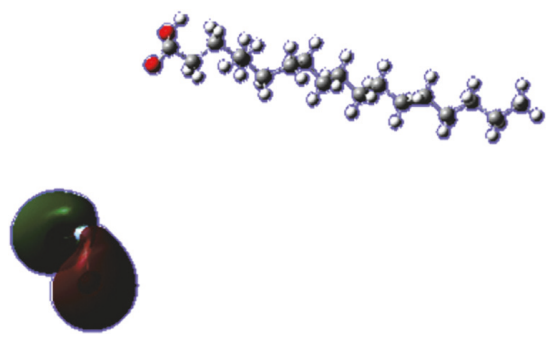

(b)

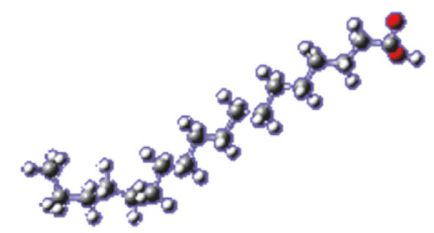

(c)

Figure 14: HOMO surfaces from molecular dynamics. Stearic acid with 3-atom silver cluster at (a) 0 ps, (b) 5 ps, and (c) 20 ps.

interactions between the nanoparticle and the biomolecule and there is not a good clearance after short periods of time.

The results illustrate that, for biological molecules, the cluster stays near 5 ps which is important in the case of introducing the coated nanoparticle in an organism because the complex has to stay stable for a minimum period of time. However, at $20 \mathrm{ps}$, the cluster moves far away from the biological molecules. This is really interesting because the intention is that once the coated molecule is introduced into the organism, the complex would be separated and it would be eliminated very easily with optimal clearance characteristics.

In order to prove that the dynamic goes well with the biomolecules studied, a file with the crystal structure of FGF Receptor 2 Tyrosine Kinase Domain Harboring the D650V Activating Mutation was chosen for the interaction with the 3 -atom silver cluster. The biomolecule was obtained from the RCSB Protein Data Bank [25].

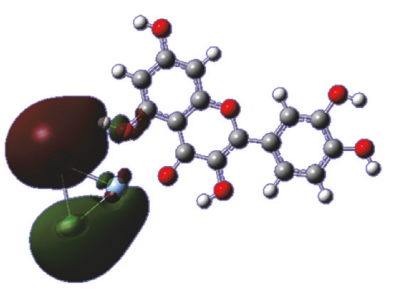

(a)

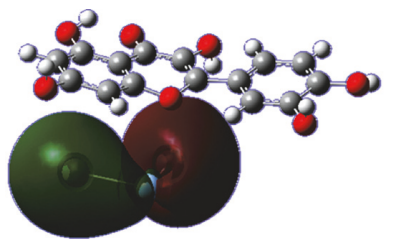

(b)
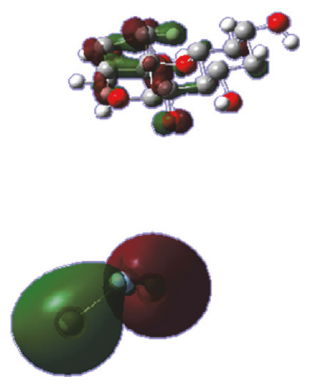

(c)

FIgURE 15: HOMO surfaces from molecular dynamics. Quercetin with 3 -atom silver clusters at (a) 0 ps, (b) 5 ps, and (c) 20 ps.

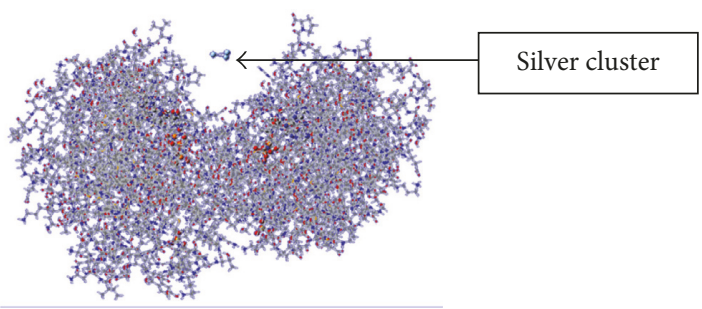

FIGURE 16: Interaction between the crystal structure of FGF Receptor 2 Tyrosine Kinase Domain Harboring the D650V Activating Mutation and the 3-atom silver cluster.

After a dynamical evolution of 5, 20, and 100 ps, there is no movement of the cluster. The system is very stable and does not move at all as shown in Figure 16. This demonstrates that there is an important difference in stability between our systems and this system.

\section{Conclusion}

A computational modeling of the silver nanoparticles and the biological molecules including glucose, stearic acid, palmitic acid, and quercetin was performed. The lowest energy conformations were identified, and the clusters of 6 silver atoms were the most stable complexes. Moreover, the study of MO describing HOMO cluster interaction contributed to find that the electronic density was around the silver cluster. Finally, molecular dynamics was performed 
using the Abalone program to observe how silver clusters interact with biological molecules for a given period of time, 5 and 20 ps. Molecular dynamics showed that, over a certain period of time, the silver cluster moved far away from the biological molecule. This means that the quercetin-coated silver nanoparticles could have good clearance properties. With respect to the interaction with the lipid layer of the skin, it can be preliminarily said that because the interaction of the silver nanoparticles with the fatty acids is not strong after a period of time, it is possible to reduce their toxicity by skin contact. In future experimental work, it would be interesting to do a full characterization of the coated silver nanoparticles and studies of cytotoxicity.

\section{Data Availability}

Data described in the manuscript are available from the authors.

\section{Conflicts of Interest}

The authors declare that they have no conflicts of interest.

\section{Acknowledgments}

Andrea Fabara would like to thank Pontificia Universidad Católica del Ecuador for all the facilities provided in the realization of their master's thesis. This research was supported by DGA-PUCE Project N13438 (2016-2017).

\section{References}

[1] R. Li, R. Chen, P. Chen, Y. Wen, P. C. Ke, and S. S. Cho, "Computational and experimental characterizations of silver nanoparticle-apolipoprotein biocorona," Journal of Physical Chemistry B, vol. 117, no. 43, pp. 13451-13456, 2013.

[2] J. Romann, J. Wei, and M. P. Pileni, "Computational matching of surface plasmon resonance: interactions between silver nanoparticles and ligands," Journal of Physical Chemistry C, vol. 119, no. 20, pp. 11094-11099, 2015.

[3] N. Lewinski, V. Colvin, and R. Drezek, "Cytotoxicity of nanoparticles," Small, vol. 4, no. 1, pp. 26-49, 2008.

[4] C. You, C. Han, X. Wang et al., "The progress of silver nanoparticles in the antibacterial mechanism, clinical application and cytotoxicity," Molecular Biology Reports, vol. 39, no. 9, pp. 9193-9201, 2012.

[5] E. Saion, E. Gharibshahi, and K. Naghavi, "Size-controlled and optical properties of monodispersed silver nanoparticles synthesized by the radiolytic reduction method," International Journal of Molecular Sciences, vol. 14, no. 4, pp. 7880-7896, 2013.

[6] S. Pal, Y. K. Tak, and J. M. Song, "Does the antibacterial activity of silver nanoparticles depend on the shape of the nanoparticle? A study of the Gram-negative bacterium Escherichia coli," Applied and Environmental Microbiology, vol. 73, no. 6, pp. 1712-1720, 2007.

[7] J. Wu, Y. Fu, Z. He et al., "Growth mechanisms of fluorescent silver clusters regulated by polymorphic DNA templates: a DFT study," Journal of Physical Chemistry B, vol. 116, no. 5, pp. 1655-1665, 2012.

[8] L. L. Landeros-Martinez, E. Orrantia-Borunda, and N. FloresHolguin, "DFT chemical reactivity analysis of biological molecules in the presence of silver ion," Organic Chemistry: Current Research, vol. 4, no. 4, pp. 1-6, 2015.

[9] J. Q. Lin, Y. G. Zheng, H. W. Zhang, and Z. Chen, "A simulation study on nanoscale holes generated by gold nanoparticles on negative lipid bilayers," Langmuir, vol. 27, no. 13, pp. 8323-8332, 2011.

[10] R. Poteau, J. L. Heully, and F. Spiegelmann, "Structure, stability, and vibrational properties of small silver cluster," Zeitschrift für Physik D Atoms, vol. 40, no. 1, pp. 479-482, 1997.

[11] S. Zhao, Z. Liu, Z. Li, W. Wang, and K. Fan, "Density functional study of small neutral and charged silver cluster hydrides," Journal of Physical Chemistry A, vol. 110, no. 40, pp. 11537-11542, 2006.

[12] Y. Santorum, Síntesis y caracterización de nanopartículas de plata empleando el extracto de las hojas de matico (Piper aduncum) como un agente reductor, Undergraduate thesis, Pontificia Universidad Católica del Ecuador, Quito, Ecuador, 2017.

[13] N. Santorum, A. Fabara, F. Pilaquinga, S. Ampudia, E. Jara, and L. Meneses, "Experimental and computational characterization of silver nanoparticles using matico (Piper aduncum) leaves extract as reducing agent," Periódico Tchê Química, vol. 15, no. 29, pp. 309-318, 2018.

[14] P. Raveendran, J. Fu, and S. L. Wallen, "A simple and "green" method for the synthesis of $\mathrm{Au}, \mathrm{Ag}$, and $\mathrm{Au}-\mathrm{Ag}$ alloy nanoparticles," Green Chemistry, vol. 8, no. 1, pp. 34-38, 2006.

[15] A. Desireddy, B. E. Conn, J. Guo et al., "Ultrastable silver nanoparticles," Nature, vol. 501, no. 7467, pp. 399-402, 2013.

[16] M. J. Frisch, G. W. Trucks, H. B. Schlegel et al., Gaussian 09, Revision A.02, Gaussian, Inc., Wallingford, CT, USA, 2009.

[17] Agile Molecule, 2006, http://www.biomolecular-modeling. com/Abalone/abalone-ii.html.

[18] Y. He and T. Zeng, "First-principles study and model of dielectric functions of silver nanoparticles," Journal of Physical Chemistry C, vol. 114, no. 42, pp. 18023-18030, 2010.

[19] H. L. Chou, C. M. Wu, F. D. Lin, and J. Rick, "Interactions between silver nanoparticles and polyvinyl alcohol nanofibers," AIP Advances, vol. 4, no. 8, p. 087111, 2014.

[20] S. Prabhu and E. K. Poulose, "Silver nanoparticles: mechanism of antimicrobial action, synthesis, medical applications, and toxicity effects," International Nano Letters, vol. 2, no. 32, pp. 1-10, 2012.

[21] C. M. Aikens, "Modelling small gold and silver nanoparticles with electronic structure methods," Molecular Simulation, vol. 38, no. 8-9, pp. 607-614, 2012.

[22] X. Chen, Y. Hu, J. Gao, Y. Zhang, and S. Li, "Interaction of melamine molecules with silver nanoparticles explored by surface-enhanced Raman scattering and density functional theory calculations," Applied Spectroscopy, vol. 67, no. 5, pp. 491-497, 2013.

[23] S. Kumar and J. K. Randhawa, "Solid lipid nanoparticles of stearic acid for the drug delivery of paliperidone," RSC Advances, vol. 5, no. 84, pp. 68743-68750, 2015.

[24] E. Lindahl, "Molecular dynamics simulations," Methods in Molecular Biology, vol. 1215, pp. 3-26, 2015.

[25] H. Berman, J. Westbrook, Z. Feng et al., "The protein data bank," Nucleic Acids Research, vol. 28, no. 1, pp. 235-242, 2000. 


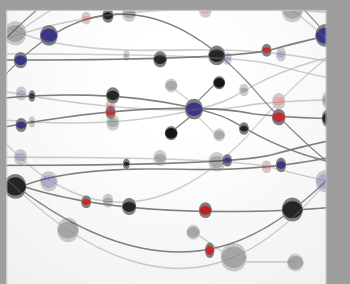

The Scientific World Journal
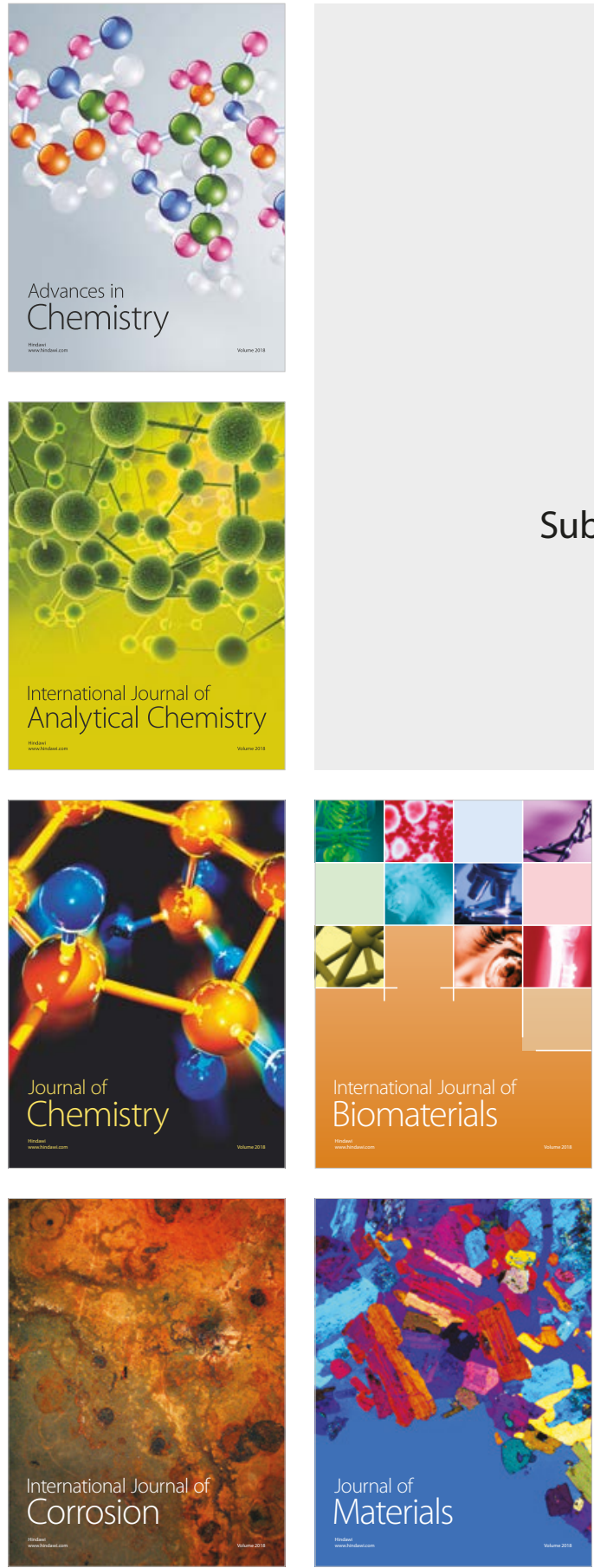

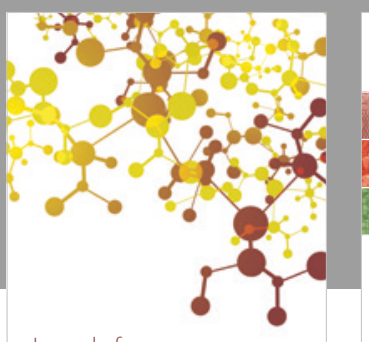

Journal of

Applied Chemistry
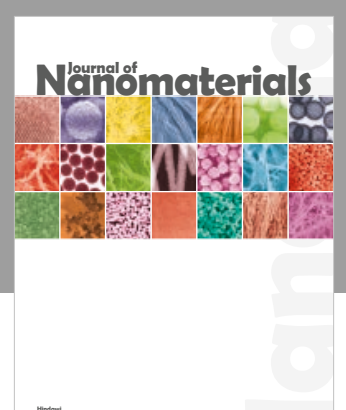

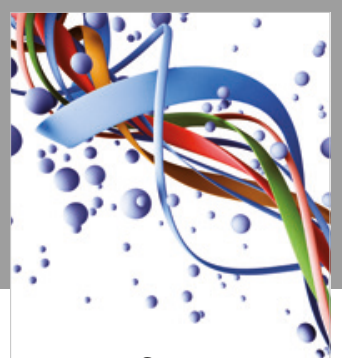

Scientifica

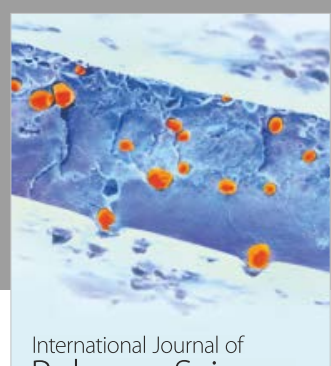

Polymer Science

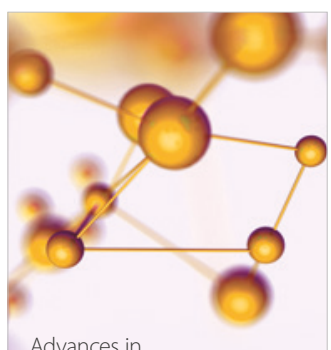

Physical Chemistry
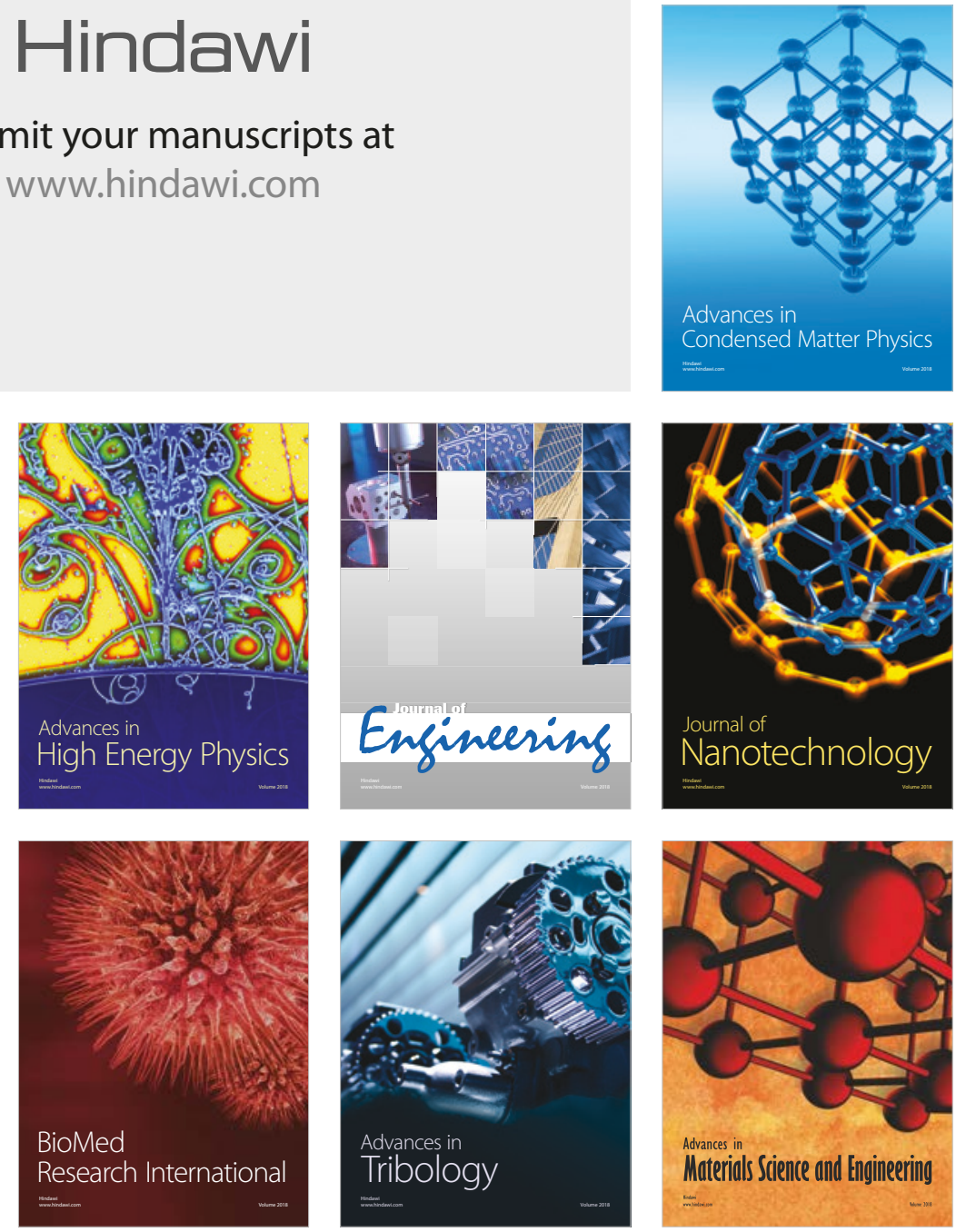